\title{
Interface shear stresses during ambulation with a below-knee prosthetic limb
}

\author{
Joan E. Sanders, PhD; Colin H. Daly, PhD; Ernest M. Burgess, MD \\ Prosthetics Research Study, Seattle, WA 98122; Center for Bioengineering, University of Washington, \\ Seattle, WA 98195
}

\begin{abstract}
Shear stresses on a residual limb in a prosthetic socket are considered clinically to contribute to tissue breakdown in below-knee amputees. When applied simultaneously with normal stresses, they can cause injury within the skin or can generate an abrasion on the surface. To gain insight into shear stresses and parameters that affect them, interface stresses were recorded on below-knee amputee subjects during walking trials. On the tibial flares, resultant shear ranged from $5.6 \mathrm{kPa}$ to $39.0 \mathrm{kPa}$, while on the posterior surface it ranged from $5.0 \mathrm{kPa}$ to $40.7 \mathrm{kPa}$. During stance phase, anterior resultant shears on a socket were directed toward the apex while posterior resultant shears were directed downward approximately perpendicular to the ground. Waveform shapes were usually double-peaked, with the first peak at $25 \%$ to $40 \%$ into stance phase and the second peak at $65 \%$ to $85 \%$ into stance. Application of these results to residual limb tissue mechanics and prosthetic design is discussed.
\end{abstract}

Key words: below-knee amputees, interface stress, prosthetic interface, resultant shear stress.

\section{INTRODUCTION}

Interface shear stresses can possibly be detrimental to a below-knee (BK) amputee in a variety of ways. Cyclic shear can cause cell separation within

\footnotetext{
Address all correspondence and requests for reprints to: J.E. Sanders, PhD, Center for Bioengineering WD-12, University of Washington, Seattle, WA 98195.
}

the skin epidermal layer, which leads to blister formation or, if the epidermis is thin, generates an open wound (1), as shown in Figure 1a. Tension can be induced when shear stresses are in opposite directions (Figure 1b), or when shear stress is generated adjacent to adherent scar tissue (Figure 1c). Skin blanching and possible cell damage result (2). Also potentially detrimental is shear stress combined with normal stress. Static shear can reduce the normal stress necessary to cause blood flow occlusion (3), as shown in Figure 1d. A concentrated normal stress adjacent to a concentrated shear stress will put the intermediate skin in tension (Figure 1e). These examples have direct application to prosthetics because it is the distribution of mechanical stress that a prosthetist controls when designing a prosthesis. Therefore, it is of vital importance to understand these stress distributions, how they change over the course of stance phase, and to which prosthetic design variables they are sensitive.

It is important to note that no etiological studies were conducted on amputee subjects to use as models for Figure 1a-e. The purpose of the figures is to illustrate loading configurations and, based on results from the literature on nonamputee subjects, their possible effects on skin.

Both normal and shear stress exist at the interface and both were measured in this research. However, in this paper, results and analysis are concentrated on shear stress. Normal stress analysis is discussed in detail elsewhere (4). 


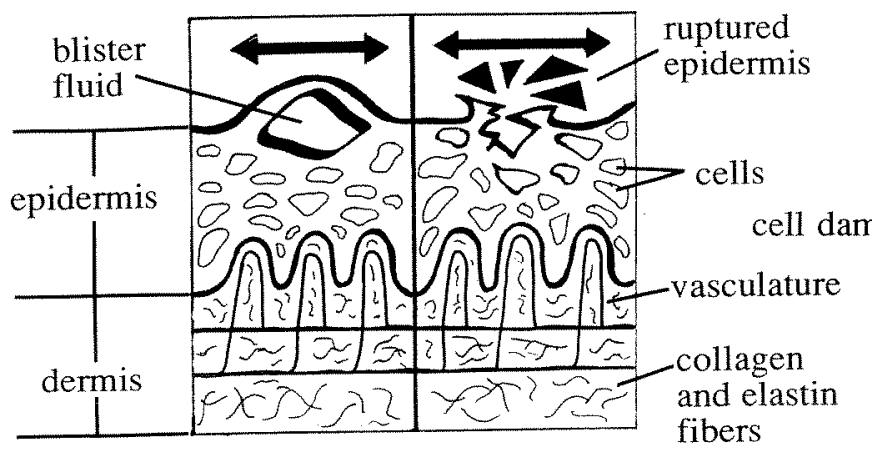

(a)

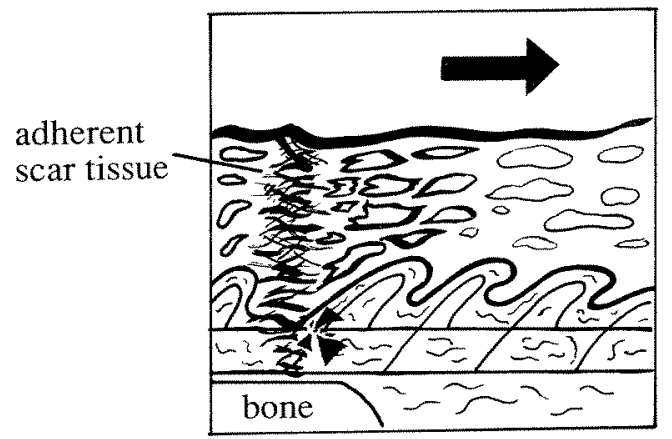

(c)

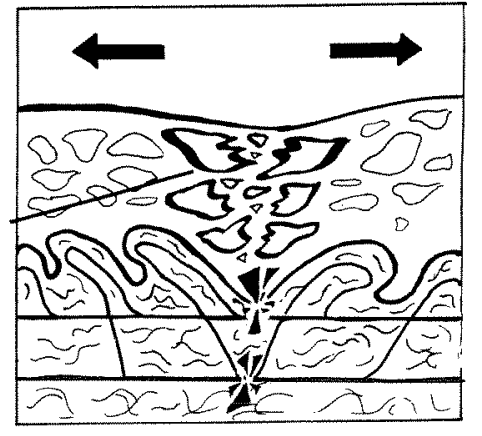

(b)

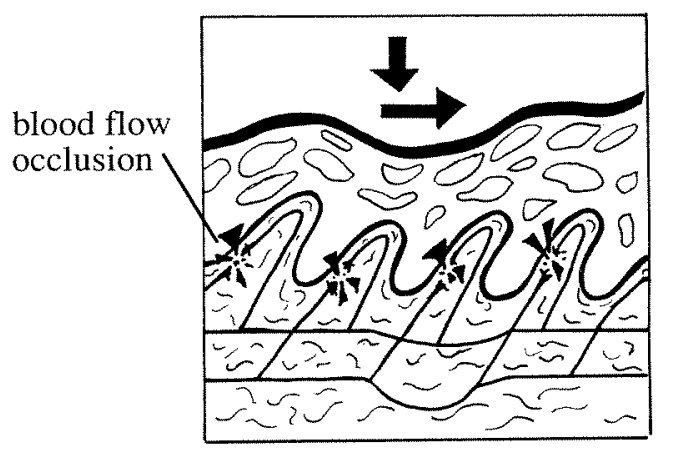

(d)

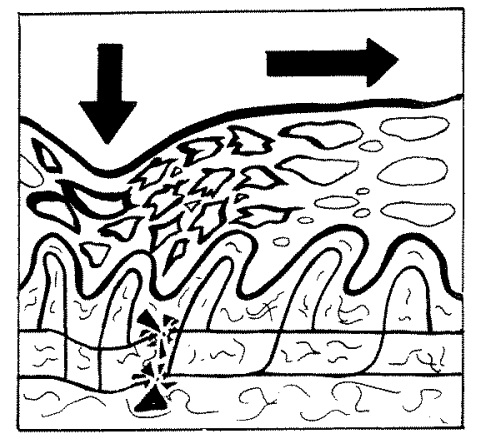

(e)

Figure 1a-e.

(a) Cyclic shear stress can cause a blister to form within the epidermis (left) or it can create an abrasion on the skin surface (right). (b) Shear stresses in opposite directions put the intermediate skin in tension, which can cause blanching and possible cell failure. (c) Shear stress adjacent to scar tissue adherent to bone puts the intermediate skin in tension. (d) Static shear stress reduced the normal stress necessary to cause blood flow occlusion. (e) A normal stress adjacent to a shear stress puts the intermediate skin in tension.

\section{BACKGROUND}

Despite their importance, interface shear stresses have not been previously measured on BK amputees. Nor has simultaneous measurement of interface stresses in three orthogonal directions been accomplished. Uniaxial shear measurement on above-knee amputees has been attempted (5) but the 


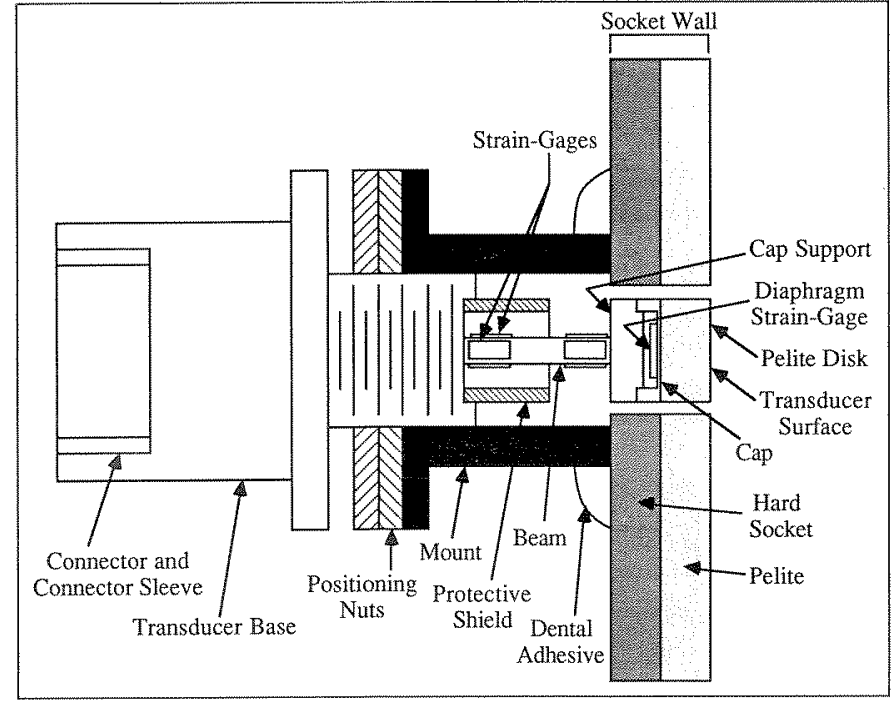

Tigure 2.

A conceptual drawing of a transducer in a mount is shown. Strain-gages on the beam were used for biaxial shear measurement and a diaphragm strain-gage was used to monitor normal stress. Leadwires (not shown) extended from the beam through holes (not shown) in the base to a connector that fit in the back of the base.

instrumentation suffered from excessive crosstalk error.

\section{METHODS}

\section{Instrumented prosthesis}

A "total-contact" patellar-tendon-bearing prosthesis was designed and fabricated for each of three BK amputee subjects. The socket and Pelite ${ }^{\mathrm{TM}}$ (KnitRite, Kansas City, MO) liner for each prosthesis were designed to be slightly smaller than that normally used by the subject since in the data collection sessions no socks or nylon sheaths were worn between the residual limb and the Pelite. Each prosthesis was completed with a Berkeley jig, an instrumented pylon (4), and a Seattle LightFoot ${ }^{\mathrm{rm}}$ (Model and Instrument Development, Seattle, WA). Suspension for each subject was: Subject \#1-a latex sleeve; Subject \#2-a latex sleeve and a suprapatellar strap; Subject \#3-a latex sleeve and a supracondylar socket. The weight of an instrumented prosthesis was $3.1 \mathrm{~kg}$, which was $1.6 \mathrm{~kg}$ more than the subjects' normal Seattle System ${ }^{\mathrm{Tm}}$ thermoplastic prosthesis, but was typical of a fitting prosthesis equipped with a Berkeley jig.

Instrumentation to measure interface stresses in three orthogonal directions during ambulation was developed and mounted to each prosthesis. Customdesigned transducers with diameters of $6.35 \mathrm{~mm}$ were positioned in mounts bonded to the outside of a socket. Transducers protruded through holes in the socket wall so that their sensing surfaces were flush with the inside Pelite liner surface. The transducer surface was a Pelite disk; no foreign material was introduced to the residual limb.

A conceptual drawing of the transducer design is shown in Figure 2. Shear measurements in two orthogonal directions in the plane of the transducer surface were achieved by mounting metal foil strain-gages on a square cross-section aluminum beam. Gages were configured in two four-arm Wheatstone bridges. Each of the two bridges measured the differences in bending moment between gage sites, which was proportional to shear force applied to the end of the transducer in a plane perpendicular to the strain-gage surfaces. This gage configuration ensured insensitivity to an off-center normal force and insensitivity to compression of the Pelite disk on the end of the transducer.

To measure stress normal to the sensing surface, a full-bridge diaphragm strain-gage was bonded to the inside face of a cap (Figure 2). Circumferential and radial gages were configured in a four-arm bridge so that bridge output was proportional to a hydrostatic pressure on the surface.

Signal conditioning and amplification for the transducers were performed by electronics housed within a backpack box carried by the subject. Amplifier gains of approximately 4,000 were used for each channel. For data storage, an $18 \mathrm{~m}$ shielded cable extended from the backpack box to a computer data acquisition unit.

Prosthetic shank forces and moments were measured simultaneously using an instrumented pylon. Instrumentation and performance characteristics for both the interface stress measurements and the shank force and moment measurements are described in further detail elsewhere (4).

During a clinical data collection session, stresses were monitored at four locations on anterior, posterior, and lateral surfaces-a total of 12 channels of data. Low socket-curvature sites of clinical interest were used. Four forces and moments in the 
Journal of Rehabilitation Research and Development Vol. 29 No. 4 Fall 1992

shank were also monitored in one of two combinations: 1) axial force, torsional moment, frontal bending moment, and sagittal bending moment, or 2) axial force, torsional moment, frontal shear force, and sagittal shear force. The total number of simultaneously monitored channels was 16 .

\section{Subjects}

All subjects were male, unilateral $\mathrm{BK}$ amputees between the ages of 23 and 46 who had suffered a traumatic injury as cause of their amputation. All were fully employed and all engaged in regular physical activity. Residual limb lengths averaged $13.7 \mathrm{~cm}( \pm 2.0)$. Each residual limb had characteristic features, but none were abnormal. Subject \#1 had a very bony residual limb with little soft tissue, especially distally, and he did not distal end-bear. Distal end-bearing was evaluated by a powdertransfer test. Subject \#2, also a non-distal endbearer with a bony residual $\lim b$, had anterolateral adherent scar tissue and a prominent medial distal osteophyte that was relieved in the socket design. Subject \#3 had excessive superficial tissue, and there were deep clefts in suture line scars at the anterodistal end. Subject \#3's socket was totalcontact and he did distal end-bear.

\section{Data collection sessions}

At least two data collection sessions were conducted on each subject. In each session, groups of at least four consecutive walking trials were conducted at each of three sagittal-plane angular alignment settings: "heel back," "zero," and "toe up." Thus, there were at least 12 trials in a session. "Zero" was optimal alignment as deemed by a team of prosthetists. "Heel back" and "toe up" were relative angular changes referenced to the center of rotation of a Berkeley jig with the axis of rotation perpendicular to the sagittal plane. Average angular values were 9 degrees of flexion and 6 degrees of extension, respectively. No linear translation was performed to accommodate foot displacement.

The pathway for walking was a $1.2 \mathrm{~m} \times 18 \mathrm{~m}$ hallway that had a concrete floor covered with vinyl tiles. A subject walked the length of the hallway in each trial, approximately eight steps, though only the central five steps were used in analysis. Walking rate was controlled to between $94 \mathrm{steps} / \mathrm{min}$ to 99 steps/min using a metronome. For all subjects walking velocity was approximately $1.3 \mathrm{~m} / \mathrm{s}$.

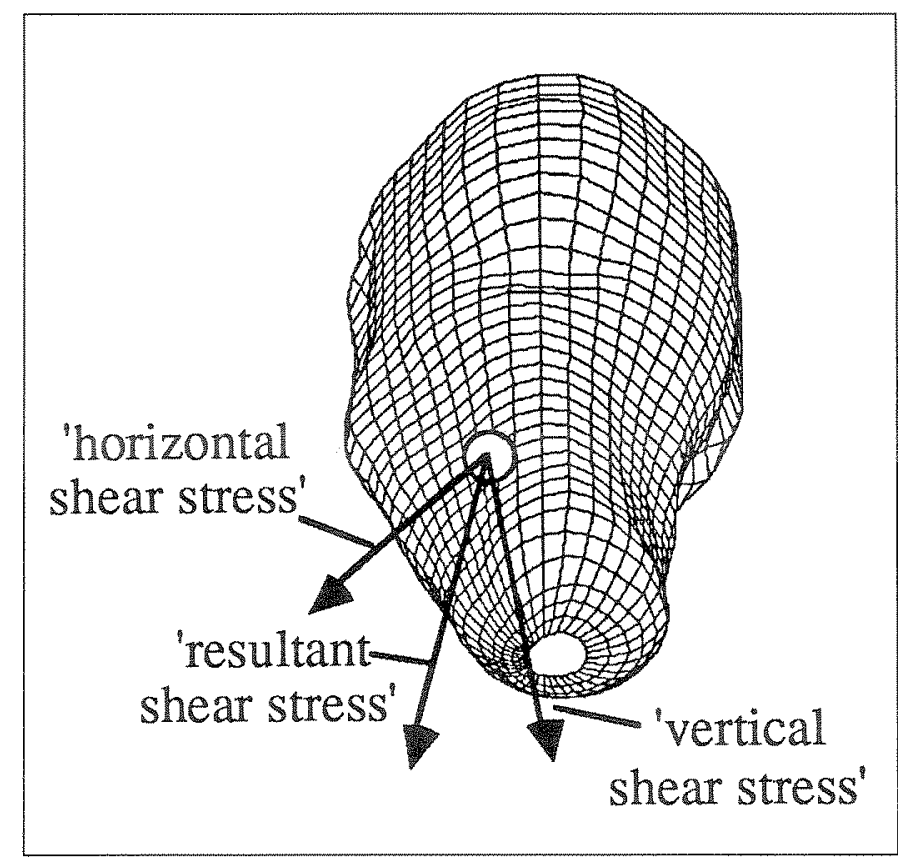

Figure 3.

Transducer shear stress sign convention is shown. At a transducer site, "horizontal shear stress" is perpendicular to "vertical shear stress." Both are in the plane of the interface. "Resultant shear stress" is the resultant vector of horizontal shear and vertical shear.

Between each group of walking trials, unloaded transducer trials and standing trials were conducted. The former were done to assess instrumentation drift over the course of a session as described in detail elsewhere (4). Unloaded transducer trials and standing trials took approximately 3 minutes each and were done while a subject was standing still.

Normal stress, resultant shear stress, and resultant shear angle at each transducer site were calculated for each step of collected data. Threshold slopes in prosthetic shank axial force waveforms of $0.63 \mathrm{~N} / \mathrm{ms}$ and $-0.63 \mathrm{~N} / \mathrm{ms}$ were used to identify heel-contact and toe-off, respectively. The 0.63 $\mathrm{N} / \mathrm{ms}$ absolute value threshold slope was selected arbitrarily, though any absolute value between 0.31 $\mathrm{N} / \mathrm{ms}$ and $1.25 \mathrm{~N} / \mathrm{ms}$ would have yielded the same results.

\section{RESULTS}

Sign conventions for results presented below are as follows: at each transducer site, normal stress is 
perpendicular to the interface and resultant shear stress is in the plane of the interface. Resultant shear stress is the resultant vector of two components: 1) "horizontal shear" is in a transverse plane and is positive in the clockwise direction when viewing a socket from above (Figure 3); and, 2) "vertical shear" is perpendicular to horizontal shear in the plane of the interface and is positive when directed downward. Stresses applied to the transducers, as opposed to stresses applied to residual limbs, are positive in sign.

All observations described below were consistent no matter which of the three alignments was considered. Analysis of changes in waveform characteristics for different alignment settings is described in detail elsewhere (4).

\section{Magnitude ranges}

Maximal interface stress magnitudes were usually achieved during the stance phase of gait. Peak magnitudes were measured in a step up to $205 \mathrm{kPa}$ for normal stress and $54 \mathrm{kPa}$ for shear stress. When averages of peak stance phase magnitudes from all steps in a session were considered, resultant shear ranged from $5.6 \mathrm{kPa}$ to $39.0 \mathrm{kPa}$ on the tibial flares, while on the posterior surface it ranged from 5.0 $\mathrm{kPa}$ to $40.7 \mathrm{kPa}$. Within a step, posteroproximal or lateral sites usually experienced the highest stresses while anteromedial proximal locations experienced the lowest stresses. Average coefficient of friction values ranged from approximately 0.01 to 0.55 and were lowest for Subject \#3, who had excessive subcutaneous fat tissue compared with Subjects \#1 and \#2.

\section{Resultant shear directions}

During approximately 20 percent to 80 percent of stance phase, resultant shear directions on anterior surfaces were inward toward the apex of the socket (Figure $\mathbf{4 a}$ and $\mathbf{4 b}$ ). The tibia was being forced anteriorly into the socket relief for the tibial crest as well as being pushed directly downward deeper into the socket. Skin over the crest of the tibia was in tension in a transverse plane.

Posterior resultant shear stresses were usually directed vertically downward approximately perpendicular to the ground (Figure $\mathbf{4 c}$ and $\mathbf{4 d}$ ). Resultant shear directions did not usually align with the socket central axis, but it is important to note that subjects tended to walk with the shank axis tilted at an angle
INTER FACE RESULTANT SHEAR MAGNITUDES AND DIRECTIONS ON SOCKET SURFACES

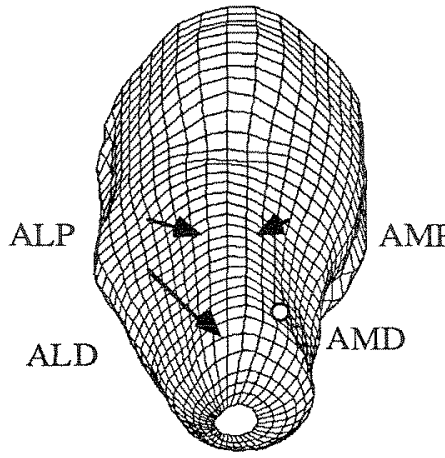

Subject \#1: Anterior Sites

(a)

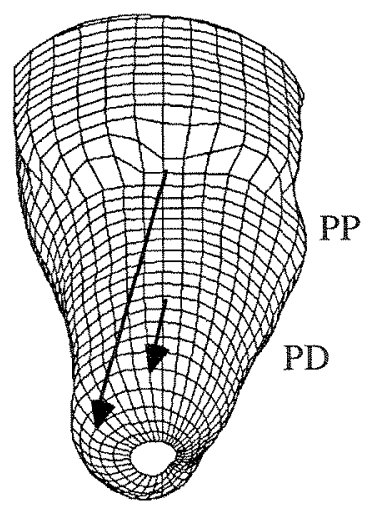

Subject \#1: Posterior Sites

(c)

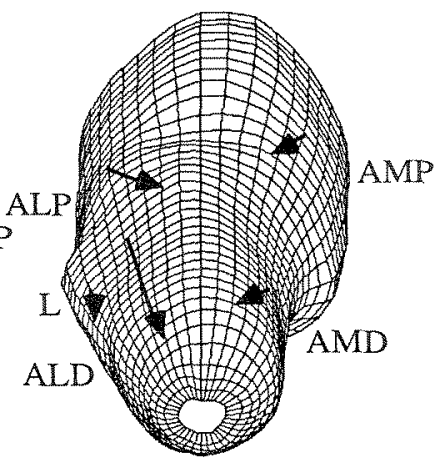

Subject \#2: Anterior Sites (b)

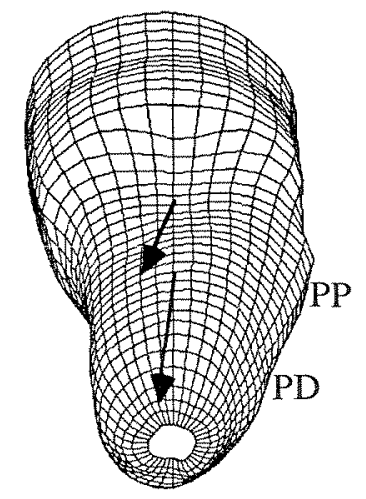

Subject \#2: Posterior Sites (d) rigure 4a-d.

Resultant shear stresses from a time $50 \%$ into stance phase are shown for Subject \#1 $(a, c)$ and Subject \#2 $(b, d)$. Arrow lengths are proportional to magnitude. ALP = anterolateral proximal; $\mathrm{ALD}=$ anterolateral distal; $\mathrm{AMP}=$ anteromedial proximal; $\mathrm{AMD}=$ anteromedial distal; $\mathrm{L}=$ lateral; $\mathrm{PP}=$ posteroproximal; $\mathrm{PD}=$ posterodistal.

other then 90 degrees with respect to the walking surface. For Subjects \#1 and \#2 the knee was medial to the foot during stance phase.

\section{Waveform shapes}

Normal stress, resultant shear stress, and coefficient of friction for a step from a trial for Subject \#1 are shown in Figure 5a-c. These results were typical of data from all subjects in the following respects:

- Waveforms were doubled-peaked, with the first peak $25-40$ percent into stance $(0.20 \mathrm{~s}$ to $0.35 \mathrm{~s}$ 
after heel contact) and the second peak at 65-85 percent $(0.51 \mathrm{~s}$ to $0.67 \mathrm{~s}$ after heel contact). Maximal stresses within a step usually occurred at the first peak except at anteroproximal sites, where they often occurred at the second peak. Interface coefficients of friction were not constant over the surface and did not show maxima at normal stress and resultant shear stress maxima.

- Resultant shear angles were unstable in approximately the first 10 percent of stance phase. An explanation is apparent in Figure 5a and $\mathbf{5 b}$. After heel contact there are time spans of minimal stress, which we have termed "loading delays," before interface stress curves begin to rise. Time spans of loading delays were not necessarily the same at all sites in a step. Usually those at posterior sites were shorter than those at anterior sites. Anteroproximal sites often were different from anterodistal sites.

- Some sites were loaded during both stance and swing phase, some only during stance, and some only during swing. In general, posterior locations remained loaded throughout the gait cycle while anterodistal sites were loaded during stance but unloaded during swing. On Subject \#3 the anterolateral proximal site was loaded during swing but unloaded during stance phase.

\section{DISCUSSION}

These results provided insight into residual-limb tissue mechanics, information which is applicable to prosthetic design and fitting.

Interface shear stresses can be of sufficiently high magnitude to cause tissue injury. In friction blister studies on anterior tibial surfaces of normals, 22 to 118 loading cycles at shear stress magnitudes of $4 \mathrm{kPa}$ to $23 \mathrm{kPa}$ with an average coefficient of friction of 0.50 were sufficient to cause breakdown (1). These are in the ranges of interface shear stresses measured here. Thus, injury shown in Figure 1a can happen. Damage illustrated in Figure 1c is possible if excessive shear is applied near adherent scar tissue.

It should be noted, however, that factors during data collection could possibly have increased shear stress magnitudes over those normally experienced by a subject. First, the subjects wore no sock in data

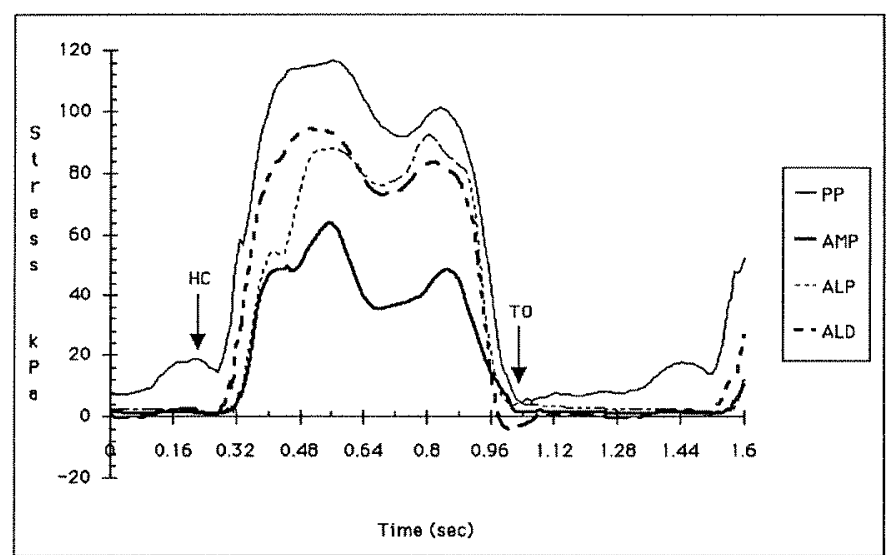

(a)

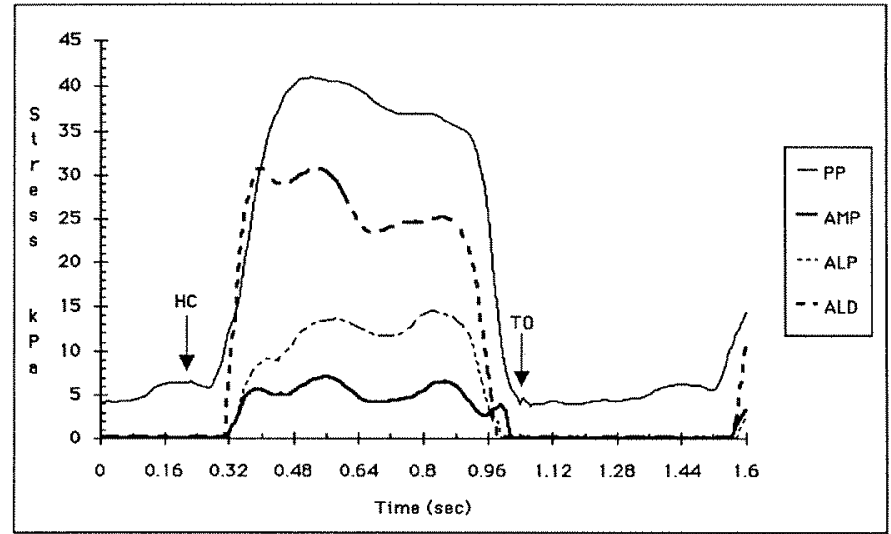

(b)

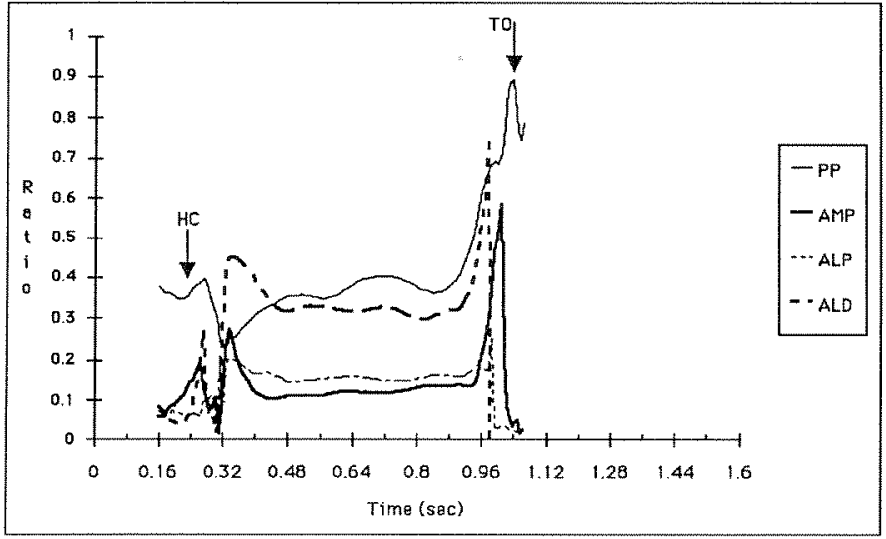

(c)

Figure 5a-c.

Stance and swing phase at "zero" alignment for a step from Subject \#1. (a) Normal stress; (b) shear stress; (c) coefficient of friction. $\mathrm{HC}=$ heel contact; $\mathrm{TO}=$ toe-off. In $(c)$ most of stance phase has been removed for clarity.

collection sessions. Second, prostheses with Berkeley jigs were used. Berkeley jigs are relatively heavy alignment devices compared with the thermoplastic devices used regularly by the subjects. Therefore, shear stress measurements collected in data collec- 
tion sessions are considered to reflect maximal levels.

Some transducer sites were not unloaded at any point during a gait cycle (e.g., the posteroproximal site in Figure 5a-c). Assuming that at those sites residual limb-prosthetic socket displacement is minimal, dynamic stresses are superposed on static stresses. Prolonged static loading has been shown to be detrimental. Daly (6) showed that blood flow can be reduced by over 90 percent when $8 \mathrm{kPa}$ normal stresses are applied on the anterior tibial surface of normals. Shear stress can reduce the pressure necessary to cause blood flow occlusion (3). Thus, vascular injury illustrated in Figure 1d may possibly occur.

Normal and shear stresses are not uniformly distributed nor are they proportionally applied. Coefficient of friction ranged from 0.01 to 0.55 . Therefore, local in-plane tension as shown in Figure 1e can be induced, which is a threat for breakdown. This is an extremely relevant clinical consideration, but one that is difficult to analyze exclusively with visual inspection techniques. It requires considering changes in local normal stress and resultant shear stress as they are dynamically applied.

Horizontal shear stresses over the crest of the tibia were directed away from the midline causing tension in the skin over the crest of the tibia. The stress shown in Figure 1b happens in combinations with normal stress. This result means that the angle of the apex of the socket over the crest of the tibia is likely to be an important design parameter in residual limb breakdown (Figure 6a and $\mathbf{6 b}$ ). An angle that matches the medial and lateral flares as in Figure 6a will distribute horizontal shear loads over a greater surface area which would induce lower horizontal stresses compared with one that does not match as in Figure 6b. However, excessive in-plane tension in skin over the tibia may be induced in $(a)$ because the skin is "hammocked" over the bone. Blanching and tissue trauma may result at a site that is not even in contact with the socket surface. The design in $(b)$ overcomes this in-plane tension problem, but it concentrates normal stresses over the tibia. This can be painful and detrimental to the tissues because the skin is very thin there. A "correct" design is between the two extremes illustrated in Figure $6 \mathbf{a}$ and $\mathbf{6 b}$. That design is one that induces a stress distribution that the particular residual limb tissues to be contained within the proposed socket will tolerate.

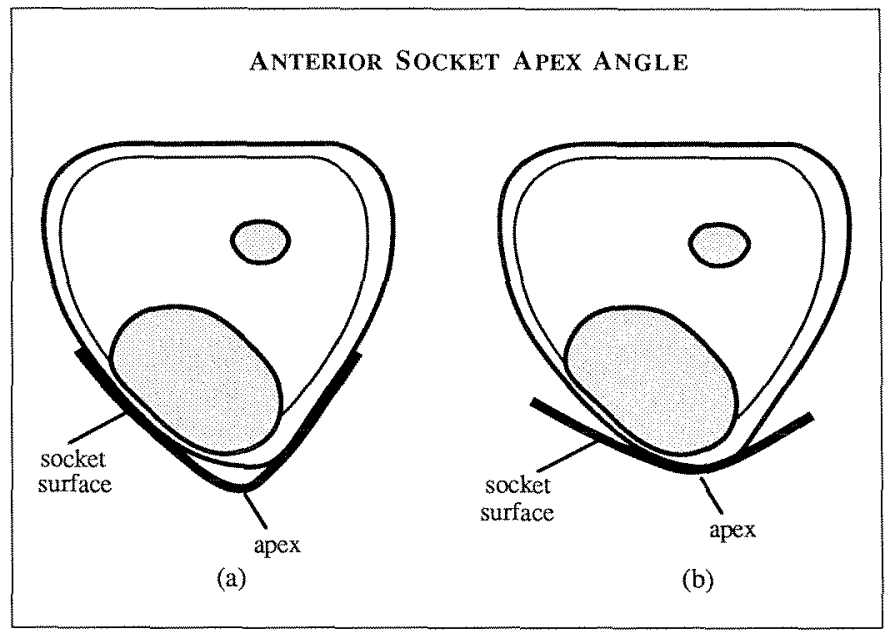

Figure 6a, b.

Two extremes of socket apex angle are shown. Each of the two designs shown ( $a$ and $b$ ) has strengths and weaknesses (see text).

Loading delays (Figure 5a and $\mathbf{5 b}$ ) may be relevant to tissue injury. A source of loading delays is slip (i.e., pistoning between the residual limb and the prosthetic socket). If due to slip, loading delays are important because of the loading configurations they may possibly induce on the anterior surface of the residual limb. Loading delays were usually not of equal time length. Thus, slip stopped at different times on the anterior surface, putting the skin under local tension in the vertical direction. Subsequently, further into stance phase, resultant shear stresses were applied principally in the horizontal direction as described above. This puts the skin under a biaxial as opposed to a uniaxial loading configuration (Figure 7a). Loading shown in Figure 1b occurs in two perpendicular directions.

Biaxial loading is important because it affects the energy absorption characteristics of skin. The stress required to produce equivalent strain is greater for skin under simultaneous tension in the perpendicular direction (7), as shown in Figure 7b. For the same internal energy change, skin experiences a higher stress if it is under biaxial rather than uniaxial load.

\section{FURTHER RESEARCH}

Further research efforts will be directed toward investigating tissue tolerance and tissue adaptation 
Journal of Rehabilitation Research and Development Vol. 29 No. 4 Fall 1992

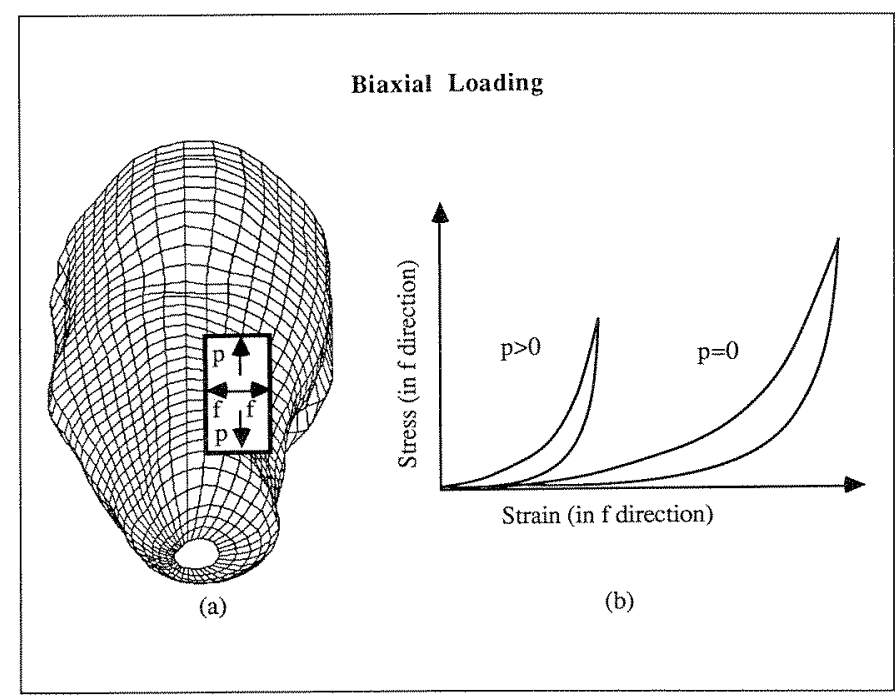

Figure 7a, b.

The stress required to produce equivalent strain is greater for skin under biaxial loading $(\mathrm{p}>0$ ) as opposed to uniaxial loading $(p=0)$.

to interface stress. The goal is to better understand what loading configurations are well-tolerated and upon what parameters tolerance and adaptation depend. That information will then be applied clinically to improve postoperative treatment strategies and to provide prosthetists with important tissue quality information applicable to fitting. Incorporation of quantitative assessment of tissue tolerance and tissue adaptation into computer-aided design software is being pursued.

\section{ACKNOWLEDGMENTS}

Support from the Whitaker Foundation; the Department of Veterans Affairs Rehabilitation Research and Development Service; and the Graduate School, Department of Rehabilitation Medicine, and Center for Bioengineering at the University of Washington is gratefully acknowledged. Assistance from Prosthetics Research Study prosthetist David Boone, CP, and artist Lori Mathews is also appreciated.

\section{REFERENCES}

1. Naylor PFD. Experimental friction blisters. $\mathrm{Br} \mathrm{J}$ Dermatol 1955;67:327-44.

2. Kenedi RM, Gibson T, Daly CH. Bio-engineering studies of the human skin II. In: Kenedi RM, editor. Biomechanics and related bio-engineering topics. Oxford: Pergamon, 1965: 147-58.

3. Bennett L, Kavner D, Lee BK, Trainor FA. Shear vs. pressure as causative factors in skin blood flow occlusion. Arch Phys Med Rehabil 1979;60:309-14.

4. Sanders JE. Ambulation with a prosthetic limb: mechanical stresses in amputated limb tissues [dissertation]. Seattle (WA): Univ. of Washington, 1991.

5. Appoldt FA, Bennett L, Contini R. Tangential pressure measurement in above-knee suction sockets. Bull Prosthet Res 1970;10(13):70-86.

6. Daly CH, Chimoskey JE, Holloway GA, Kennedy D. The effect of pressure loading on the blood flow rate in human skin. In: Kenedi RM, Cowden JM, Scales JT, editors. Bedsore biomechanics. London: MacMillan, 1976: 69-77.

7. Lanir Y, Fung YC. Two-dimensional mechanical properties of rabbit skin II: experimental results. J Biomech 1974;7:171-82. 